\title{
A cluster-randomized trial to reduce major perinatal morbidity among women with one prior cesarean delivery in Québec (PRISMA trial): study protocol for a randomized controlled trial
}

\author{
N. Chaillet ${ }^{1,11^{*}}$, E. Bujold ${ }^{1}$, B. Masse ${ }^{2}$, W. A. Grobman ${ }^{3}$, P. Rozenberg' ${ }^{4}$, J. C. Pasquier ${ }^{5}$, A. Shorten ${ }^{6}$, M. Johri ${ }^{7}$, \\ F. Beaudoin ${ }^{8}$, H. Abenhaim', S. Demers ${ }^{1}$, W. Fraser ${ }^{5}$, M. Dugas ${ }^{10}$, S. Blouin ${ }^{1}$, E. Dubé ${ }^{1}$, R. Gauthier ${ }^{8}$ and PRISMA Trial \\ Research Group
}

\begin{abstract}
Background: Rates of cesarean delivery are continuously increasing in industrialized countries, with repeated cesarean accounting for about a third of all cesareans. Women who have undergone a first cesarean are facing a difficult choice for their next pregnancy, i.e.: (1) to plan for a second cesarean delivery, associated with higher risk of maternal complications than vaginal delivery; or (b) to have a trial of labor (TOL) with the aim to achieve a vaginal birth after cesarean (VBAC) and to accept a significant, but rare, risk of uterine rupture and its related maternal and neonatal complications. The objective of this trial is to assess whether a multifaceted intervention would reduce the rate of major perinatal morbidity among women with one prior cesarean.

Methods/design: The study is a stratified, non-blinded, cluster-randomized, parallel-group trial of a multifaceted intervention. Hospitals in Quebec are the units of randomization and women are the units of analysis. As depicted in Figure 1, the study includes a 1-year pre-intervention period (baseline), a 5-month implementation period, and a 2 -year intervention period. At the end of the baseline period, 20 hospitals will be allocated to the intervention group and 20 to the control group, using a randomization stratified by level of care. Medical records will be used to collect data before and during the intervention period. Primary outcome is the rate of a composite of major perinatal morbidities measured during the intervention period. Secondary outcomes include major and minor maternal morbidity; minor perinatal morbidity; and TOL and VBAC rate. The effect of the intervention will be assessed using the multivariable generalized-estimating-equations extension of logistic regression. The evaluation will include subgroup analyses for preterm and term birth, and a cost-effectiveness analysis.

(Continued on next page)
\end{abstract}

\footnotetext{
* Correspondence: nils.chaillet@fmed.ulaval.ca

'Department of Obstetrics and Gynaecology, Laval University, Quebec, QC, Canada

${ }^{11}$ Faculté de Médecine, Département d'Obstétrique \& Gynécologie,

Université Laval, Centre de recherche du CHUQ, 2705, Boul. Laurier, local

T-R-92, Quebec, QC G1V 4G2, Canada

Full list of author information is available at the end of the article
} 
(Continued from previous page)

Discussion: The intervention is designed to facilitate: (1) women's decision-making process, using a decision analysis tool (DAT), (2) an estimate of uterine rupture risk during TOL using ultrasound evaluation of low-uterine segment thickness, (3) an estimate of chance of TOL success, using a validated prediction tool, and (4) the implementation of best practices for intrapartum management.

Trial registration: Current Controlled Trials, ID: ISRCTN15346559. Registered on 20 August 2015.

Keywords: VBAC, Uterine rupture, Intrapartum management, RCT

\section{Background}

Compared to historical rates, the rate of cesarean deliveries is high in industrialized countries. In Canada, these rates increased from 21.2 to $28.0 \%$ between 2000 and 2008 and remained stable since 2011 [1-3]. Previous cesarean deliveries alone are an indication for over $30 \%$ of all cesareans in Canada. Every year, over 30,000 women in Canada who have undergone a cesarean delivery will be faced with a difficult choice for their next pregnancy, i.e.: (1) plan for a second cesarean delivery, associated with a higher risk of maternal complications than a vaginal delivery $[4,5]$, or (2) have a trial of labor (TOL) to achieve a vaginal birth after cesarean (VBAC) and accept a significant, but rare, risk of uterine rupture [6-9], which constitutes a principal complication related to a TOL [10-16]. However, although an elective repeat cesarean (ERC) may prevent uterine rupture in most cases, this intervention is associated with a higher risk of maternal and perinatal complications, particularly compared to a VBAC $[4,16-21]$. The choice of the mode of delivery among women with a prior cesarean delivery constitutes an important public health issue within the current context, in which health professionals hesitate, due to a variety of reasons - including the risk of litigation - to recommend a TOL in the absence of validated and effective methods that can predict the risk of uterine rupture [22, 23].

Recently, new clinical tools have been developed to assist in women's decision-making [24], including those that help estimate the chance of TOL success [25-27], and the risk of uterine rupture [16, 28-30]. Several RCTs performed in pregnant women and a recent meta-analysis [24], demonstrated that decision analysis tools (DAT) are effective in improving women's knowledge; reducing anxiety and decisional conflict; and guiding women's final choice during the decisionmaking process [31-44]. Based on a study involving 9616 women attempting VBAC in United States, Grobman et al. developed a nomogram to assess the chances of TOL success based on antepartum clinical factors and factors available upon admission at the time of delivery $[25,26]$. Two observational studies and the recent statements of the NIH Consensus Development
Conference confirmed the validity of this instrument in North America [16, 45-47]. Grobman et al. also highlighted that a prediction model for TOL success provides also additional information regarding the chance of TOL-related morbidity. They showed that maternal and neonatal morbidity is not greater for those women who undergo TOL than for those who undergo an elective repeat cesarean delivery when the chances of VBAC success are at least 70\% [48]. Finally, two observational studies in Québec, along with a meta-analysis, assessed the effectiveness of measuring the lower uterine segment thickness (LUS) by ultrasound between the 35 and 38 week of pregnancy, to estimate the risk of uterine rupture [28, 29, 49]. Findings indicated: (1) the combination of abdominal and endovaginal ultrasounds allowed an optimal detection rate for uterine scar defect; $[50,51],(2)$ a LUS thickness $<2.0 \mathrm{~mm}$ represent a higher risk of uterine rupture ( $>1 \%$ ), a LUS thickness $\geq 2.0$ and $<2.5 \mathrm{~mm}$ represent a moderate risk of uterine rupture (around 0.5\%), and a LUS thickness $\geq 2.5 \mathrm{~mm}$ represent a low risk of uterine rupture $(<0.3 \%)$; [49] and (3) the use of LUS thickness in clinical practice was associated with no symptomatic uterine rupture among 984 women who underwent a TOL after one prior cesarean delivery [49], compared to $0.36 \%$ in the meta-analysis of Guise et al. [16]. These results were confirmed by a recent meta-analysis on the effect of the LUS method on uterine rupture $(\mathrm{OR}=0.19$; CI $95 \%=0.09$ to 0.40 ) [29], supporting the hypothesis that the LUS method, performed between the 35 and 38 week of pregnancy, is currently the most effective instrument to predict uterine rupture in women with previous lowtransverse cesarean $[28,29,49]$.

The availability of new, validated and standardized tools may facilitate the decision-making process as they may assist in the prediction of TOL success as well as of uterine rupture $[24,28,29,49]$. However, their effectiveness in leading to an optimal selection of women for a TOL remains uncertain. This trial was designed to assess whether a multifaceted intervention based on the implementation of these tools into clinical practice (PRISMA program) will reduce the rate of major neonatal morbidities among women with one prior cesarean delivery. 


\section{Hypothesis}

Our primary hypothesis is that women exposed to the PRISMA program (Process for decision-making, RISk assessment and MAnagement in obstetrics) will have a rate of major perinatal morbidity, as measured in the hospitals during the intervention period, that is reduced by $25 \%$ in comparison with the control group (relative reduction). Secondary hypotheses are that the PRISMA program will: (1) reduce the rate of major maternal morbidity, (2) reduce the rate of minor maternal and perinatal morbidity, and (3) increase the rate of VBAC.

\section{Methods/Design}

\section{Study design}

This is a non-blinded, multicentre, stratified, clusterrandomized, parallel-group trial in which hospitals are the units of randomization and patients are the units of analysis. Randomization will be stratified according to level of care (community, regional, or tertiary hospital). The study included a 1-year pre-intervention (baseline) period, a 5-month implementation period, and a 2-year intervention period (Fig. 1).

\section{Inclusion and exclusion criteria}

All hospitals entered the trial in April 2016. The study is conducted in 40 out of 48 eligible public hospitals in the province of Québec. To be eligible, each center was required to offer TOL after cesarean in their population; to have a minimum of 300 deliveries in the previous year; to have functional operating rooms; to have access to a high-performance sonographic device; and at the time of recruitment, to have no recent or ongoing quality-improvement programs specifically designed to target women with one prior cesarean delivery. Hospitals where LUS thickness measurement was already available in routine clinical practice and will remain available during the study were excluded from the trial.

At the patient level, women with only one prior cesarean delivering at one of the participating centers at a minimum of 24 weeks with one newborn who weighs at least $500 \mathrm{~g}$ at delivery are included in the analyses. Women with delivery or miscarriage before 24 weeks of gestation, with more than one previous cesarean delivery or with multiple gestations will be excluded from the trial.

\section{Intervention group activities}

Intervention group activities will start in April 2017 and will end in August 2019. The PRISMA program, which will be implemented at the hospital level in the intervention group, targets physicians and nurses involved in the decision-making process for an ERC or a TOL among women with one prior cesarean delivery. The PRISMA program consists of: (1) a decision-aid tool to support women's decision making in the choice of the mode of delivery (DAT), (2) standardized risk assessment tools leading to an optimal selection of women eligible for a TOL, i.e.: (a) an estimation of the chance of vaginal birth after cesarean (VBAC) success (Grobman nomogram), and (b) an estimation of the risk of uterine rupture using ultrasound measurement of lower uterine segment (LUS) thickness, and (3) a professional training program from the Society of Obstetricians and Gynaecologists of Canada (SOGC) to describe an optimal management of delivery and labor dystocia for a TOL. No financial incentive will be provided.

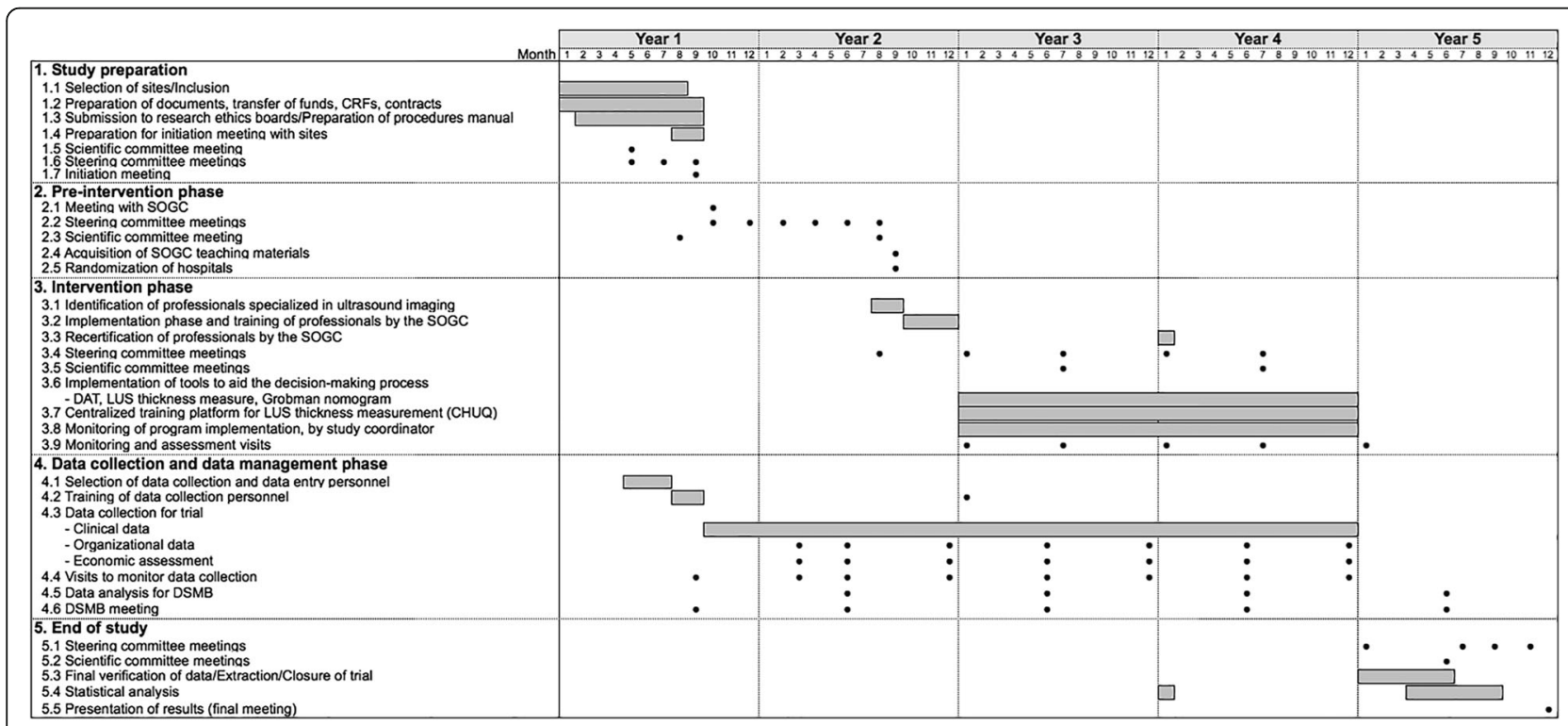

Fig. 1 SPIRIT schedule for trial preparation, pre-intervention, intervention and assessments 


\section{Professional training program from the SOGC}

The 5-month implementation period will focus on implementing the PRISMA program in each of the 20 hospitals randomized to the intervention group. In each centre receiving the intervention, health professionals in obstetrics (physicians, nurses qualified in obstetrics, and midwives, if available) will be invited to participate in an onsite 1-day training workshop, provided by certified instructors from the Society of Obstetricians and Gynaecologists of Canada (SOGC), on best practices regarding intrapartum management among women with one prior cesarean delivery, as well as on the PRISMA clinical prediction tools. Using SOGC clinical practice guidelines and algorithms, health personnel will be trained on the following topics: prolonged pregnancy, management of the first and the second stages of labor, induction and stimulation of labor, fetal health surveillance, use of the WHO partograph [52], and assisted vaginal delivery. The SOGC training program will also include a workshop on the criteria to be assessed and discussed with the women during the decision-making process, as well as on the clinical use and interpretation of the Grobman prediction tool and of the ultrasound evaluation of the risk of uterine rupture. The training program will also sensitize participants to maternal factors contributing to the choice of the mode of delivery. Finally, in each hospital receiving the intervention, one or two technicians and physicians, with expertise in sonography, will be identified and invited to receive additional 1-day training in sonographic techniques related to the evaluation of the risk of uterine rupture. A half-day of recertification will be scheduled at the beginning of the second year of the intervention period. The entire training program will be overseen by the SOGC, which possesses extensive expertise in the field of professional training [53]. Details of the training program and algorithms will be available after the trial (September 2019) upon request by email to the corresponding author (NC).

\section{Implementation of clinical tools leading to an optimal selection of women eligible for a TOL}

After the 5-month implementation period, physicians from the intervention hospitals will be asked to use the three clinical tools of the program that assess the women's initial preferences (DAT), the risk of uterine rupture (LUS) and the chances of TOL success (Grobman nomogram) for a period of 2 years from September 2017 to August 2019 (intervention period).

Decision analysis tool The DAT used in this trial, entitled "Giving Birth after Cesarean Section: Making an Informed Choice", has been adapted from the A. Shorten Birth Choices study [43], validated in Quebec in 2011 [54], and has received a 96\% ranking on the
International Patient Decision Aid Standard (IPDAS) assessment scale $[55,56]$. The DAT is divided into two sections: (A) an Information section, providing a description and an explanation of the risks and benefits of each option, and (B) an Exercise section, allowing women to clarify and summarize their values and preferences with their physician about the choice of the mode of delivery, indicating what suggestions are important and how important these suggestions are (not important, important, very important). The DAT will be distributed between $12^{0 / 7}$ and $34^{6 / 7}$ weeks of pregnancy by the treating physician, preferentially early in the pregnancy, to all women with one prior cesarean delivery during their follow-up visit. The physician will ask the women to read the DAT carefully and complete the Exercise section, so that they will be able to discuss their preferences during their next appointment.

Grobman nomogram Antepartum assessment of the chances of TOL success among women with one prior cesarean delivery will be conducted by the treating physician between $12^{0 / 7}$ and $34^{6 / 7}$ weeks of pregnancy, during one of the patient's follow-up visits [25]. The treating physician will use the Grobman nomogram, derived from logistic regressions, which generates probability of VBAC success and a confidence interval of $95 \%$ [25-27, 46, 47]. This tool is based on maternal characteristics available at the time of the first prenatal visit, i.e. maternal age, Body Mass Index, prior vaginal delivery, prior successful VBAC, and recurrence of indication of prior cesarean delivery [25]. The assessment will then be completed after $36^{6 / 7}$ weeks of pregnancy, according to the maternal characteristics available at the time of admission, i.e.: estimated gestational age at delivery; hypertensive disease of pregnancy; labor induction; effacement; dilation; and station [26]. Results related to VBAC success and risk level will be discussed with each woman during their pregnancy and upon admission for delivery.

Ultrasound assessment of the risk of uterine rupture (LUS clinic) The treating physician will routinely refer eligible women to the LUS clinic between $35^{0 / 7}$ and $38^{6 / 7}$ weeks of pregnancy. Each woman will meet with a nurse who will verify her eligibility, and will obtain her consent to the LUS. Eligibility criteria for the sonogram include: one prior cesarean delivery, and no uterine incision or laceration other than lower transverse; no multiple gestation; no known lethal congenital abnormalities; no preterm rupture of membranes; and no labor at the time of sonography. After ensuring that the woman has a full bladder, a trained ultrasound technician or a physician will measure the lower uterine segment (LUS) thickness by abdominal 
and endovaginal ultrasounds. The lower uterine segment will be swept in a sagittal cut from the right extremity to the left extremity, with the probe placed to detect the thinnest area of the lower segment. The technician/physician will also record the amniotic fluid index, the biparietal diameter, the cranial and abdominal circumference and the femoral length, in order to estimate the fetal weight. For each of the abdominal and endovaginal ultrasounds, the technician/ physician will capture three pictures and a 15-s film (2D-ultrasound in real time).

Each 15-s film will be then immediately sent to a centralized platform in Québec in order to allow an external validation of each film in less than $1 \mathrm{~h}$ by experienced experts in measurement of the LUS thickness. This continuous training platform will accelerate the learning curve among health professionals in hospitals from the intervention group. The technicians of the centralized platform will capture three total measurements of the thinnest lower segment for each of the abdominal and endovaginal ultrasounds, based on the two 15-s films, and then send the results to the physician of the LUS clinic at the referring hospital. Once the measurements have been taken and the results of the external validation in Québec are available, the physician responsible of the LUS clinic, who has also been trained, will validate the exam and reproduce it immediately, as required. Only the thinnest measurement, from among the six measurements taken via the abdominal and endovaginal ultrasounds, will be used. For each professional, a continuous quality assessment of the measurement, based on objective criteria, will be conducted during the first 30 measurements of the LUS thickness. Additional training could be considered depending on the performance of each professional $[28,50,51,57-59]$.

The physician in charge of the LUS clinic will complete a consultation form to be sent to the treating physician. The treating physician will be informed of the estimated fetal weight (EFW) and the risk of uterine rupture and will discuss the results with the woman. According to lower uterine segment thickness, women will be classified into three risk categories for uterine rupture: high risk $(<2.0 \mathrm{~mm})$, intermediate risk $(\geq 2.0 \mathrm{~mm}$ and $<2.5 \mathrm{~mm})$, and low risk $(\geq 2.5 \mathrm{~mm})$. If the EFW falls below the 95th percentile and the LUS thickness is $\geq 2.5 \mathrm{~mm}$, the treating physician will be informed that the risk of uterine rupture is $<0.3 \%$ in the absence of labor induction with unfavorable cervix, and that a TOL should be recommended in the absence of other contraindications $[16,28,29,45,60-62]$. If the LUS thickness is $<2.0 \mathrm{~mm}$, the treating physician will be informed that the risk of uterine rupture is $>1 \%$, and that a TOL should not be considered except in exceptional circumstances (e.g. admission at full cervical dilatation).
If the LUS thickness is $\geq 2.0$ and $<2.5 \mathrm{~mm}$, the treating physician will be informed that the risk of uterine rupture is intermediate (around 0.5\%), and that a TOL could be considered if no other particular conditions are observed. The exact LUS thickness, however, will not be conveyed to the patient or treating physician.

\section{Control group}

No intervention from the PRISMA team is planned for the control group. In order to assess contamination bias, quality-improvement programs will be reviewed annually in control hospitals.

\section{Study outcomes}

The primary outcome is the major perinatal morbidity rate. The composite risk of major perinatal morbidity includes: (1) In utero, intrapartum and neonatal death, defined as the fetal death in utero, during labor or prior to 28 days of age (excluding lethal congenital abnormalities); (2) APGAR score at $5 \mathrm{mn}<4$; (3) Metabolic acidosis (umbilical arterial $\mathrm{pH}<7+$ base excess $\leq-12 \mathrm{mmol} /$ l); (4) Major trauma (skull fracture, subdural/subarachnoid haemorrhage, brachial plexus injury, spinal cord injury, major genital injury, paresis/paralysis at discharge); (5) Intracerebral/intraventricular haemorrhage (grades 3 and 4); (6) Periventricular leukomalacia; (7) Seizure (occurring from delivery to discharge); (8) Invasive mechanical ventilation with endotracheal intubation; (9) Major respiratory morbidity (bronchopulmonary dysplasia treated with oxygen or ventilation at 36 weeks post menstrual age or at 28 days of life, persistent pulmonary hypertension of the newborn, pneumothorax, pulmonary haemorrhage, hyaline membrane disease requiring mechanical ventilation); (10) Necrotising enterocolitis, stage 2 and 3 ; 11) Hypoxic-ischemic encephalopathy (APGAR $5 \mathrm{mn}<4+\mathrm{pH}<7+$ base excess $<-12 \mathrm{mmol} / \mathrm{L}$ + seizure); (12) Proven neonatal sepsis/infection (positive blood or cerebrospinal fluid culture); and (13) Hypotension requiring vasopressor support. The binary primary outcome $(0 ; 1)$ event is triggered by occurrence any of the above morbidities. Value 1 is attributed if at least one of the above conditions is met.

Secondary outcomes include:

1. The rate of major maternal morbidity: this indicator is measured with a composite factor of maternal morbidity including: (1) maternal death $(\leq 6$ weeks postpartum); (2) hysterectomy; (3) proven thromboembolic complications (thrombophlebitis, pulmonary emboli); (4) admission to intensive care unit for $\geq 4$ days; (5) acute pulmonary edema/ cardiogenic shock; (6) sepsis with proven bacteremia (septicemia); (7) injury/lacerations to internal organs, requiring surgical repair (bladder, bowel, 
large vessels); (8) symptomatic uterine rupture (complete rupture of the uterine wall with extrusion of amniotic fluid, placenta, or fetal limb outside the uterine cavity, or with rupture of the bladder, requiring surgical repair)

2. The rate of minor maternal morbidity: this indicator is measured with a composite factor of maternal morbidity including: (1) blood transfusion; (2) postpartum hemorrhage requiring surgical procedure; (3) perineal tear of third or fourth degree; (4) cervical lacerations requiring repair; (5) dehiscence of skin wound (C-section or perineal); (6) puerperal fever ( $\geq 38{ }^{\circ} \mathrm{C}$ postpartum); (7) postpartum infection requiring drugs or surgery (infection of the incision (C-section or perineal), uterus (e.g. endometritis), urinary tract $\left(\geq 10^{5} \mathrm{CFU} / \mathrm{ml}\right)$, respiratory tract (e.g. pneumonia)); (8) major gastrointestinal complications (intestinal occlusion); (9) obstetric anesthesia complications (neurological, cardiovascular, respiratory); (10) length of postpartum hospital stay $\geq$ 7 days; (11) admission to intensive care unit for less than 4 days; and (12) readmission to hospital within 40 days of childbirth

3. The rate of minor perinatal morbidity: this indicator is measured with a composite factor of perinatal morbidity including: (1) minor cardiorespiratory complications (cardiac rhythm disorders, delayed amniotic fluid clearance, transient tachypnea of the newborn); (2) non-invasive mechanical ventilation; (3) admission to the intensive care unit for less than 4 days; (4) oxygen therapy; (5) suspicion of neonatal infection (culture-negative); (6) APGAR score $\geq 4$ and $<7$ at $5 \mathrm{~min}$; (7) acidosis (umbilical artery $\mathrm{pH} \geq 7$ and $<7.20$ ); (8) minor trauma (lacerations, limb fracture, clavicle fracture); (9) transfusion in first $24 \mathrm{~h}$; and (10) weight loss $>10 \%$ in first ten postnatal days

4. Rate of trial of labour (TOL): this is the number of women attempting a vaginal delivery divided by the total number of women with one previous $\mathrm{C}$-section

5. Rate of $V B A C$ : this is the number of vaginal deliveries divided by the total number of women with one previous cesarean delivery

\section{Randomization and allocation}

In April 2017, after the 1-year baseline period, hospitals will be randomly assigned either to the intervention group or the control group. Randomization will be stratified according to level of care of each hospital (community, regional, or tertiary hospital). To avoid imbalance in the size of the two groups, we will use computer-generated, blocked randomization within each stratum, with blocks consisting of four centers or, for strata with fewer than eight hospitals, two centers. The names of participating hospitals will be coded and the randomization will be generated by an independent statistician blinded to group allocation. The Data Safety Monitoring Board will receive the results of the randomization and the key code to ensure validity of the process and reveal the distribution of both intervention and control groups to the coordination team. Local investigators at each hospital will then immediately be informed of the assignment status of their hospital.

\section{Data collection and management Data collection}

Clinical data are collected independently of the intervention. Subjects will be initially screened by the medical archives department staff in every participating hospital, and then will be validated by the data collectors according to the study inclusion and exclusion criteria. Computerized data collection will be performed on-site using Dacima Clinical Suite from Dacima Software Inc. [63] The electronic case report form includes individual clinical information about the mother and the newborn, namely maternal characteristics, prenatal care, labor and delivery and maternal and neonatal complications. Inhospital data will be abstracted by trained research nurses or medical archivists from the medical records of mothers and newborns at least 3 months after delivery; data will be abstracted in the same way at both intervention and control hospitals from April 2016 to March 2017 (baseline period) and from September 2017 to August 2019 (intervention period). Data collectors will be aware of the randomization assignments but will not be involved in outcomes assessment.

\section{Data management}

The Dacima Clinical Suite and the trial database are managed by the Data Management Team located at the Research Center of the CHU de Québec (Laval University, Québec City, QC) and hosted on secure servers in Dacima software facilities (Montreal, QC). Until the end of the trial, access to the clinical database will be restricted to the Data Management Team. All steps involved in the management of clinical data will be annually monitored by an independent Data Safety Monitoring Board (DSMB).

\section{Quality control of data}

Four levels of control have been set up. The first level represents the data entry controls included in the Dacima software (bounds, branching logics, associations between variables). The second level is a random case review of around 5\% of all medical records by a second data collector. In addition, all cases of major perinatal and maternal morbidity will be systematically monitored for quality. The third quality control level targets specific cases of perinatal and maternal morbidity for which a 
consensus between the collectors and the data management team could not be reached. These cases will then be submitted to a committee of experts (obstetricians and neonatologists) for further study. Finally, the Data Management Team will assess the data completeness and quality directly in the database (fourth level of control). Discrepancies will be resolved through onsite visits and queries sent to data collectors.

\section{Statistical analysis plan}

\section{Sample size and power calculation}

The sample size was calculated to maximize statistical power while minimizing the number of clusters [64]. Sample size calculations were based on a cluster RCT design [65], stratified by level of care (community, regional or tertiary care hospitals), to detect a relative reduction of $25 \%$ in major perinatal morbidity in hospitals of the intervention group compared with those of the control group. To account for clustering by hospital, we assumed an intraclass correlation coefficient of 0.001, estimated from the QUARISMA Trial data, representing $70 \%$ of deliveries in Quebec in 2010-2011 [64]. We calculated that we would have to enroll 40 hospitals in the study, with an expected total of 7360 women with one prior cesarean delivery per year $(22,080$ women for the 1-year baseline period and the 2-year intervention period), to achieve a power of $80 \%$ to detect a $25 \%$ relative reduction with the intervention in the major perinatal morbidity rate, assuming a baseline rate of $4.5 \%$ for the three strata, at a two-sided alpha significance level of $0.05[64,66]$.

\section{Type of analysis and handling loss-to-follow-up}

Analyses will be conducted in intention-to-treat, i.e., each patient will be analyzed in the hospital to which she will be admitted and each hospital will be analyzed in the group to which it will be assigned by the randomization. If a hospital decides to withdraw from the trial after the randomization, data collection will continue until the end of the trial, according to the commitment made by the hospital authorities at the time of inclusion. This hospital will not be excluded from the analyses.

\section{Statistical analysis}

For reducing contamination bias, hospitals are the units of randomization and patients are the units of analysis $[64,67]$. In the primary intention-to-treat analyses, we will assess the effect of the intervention on the rate of major perinatal morbidity using the multivariable generalized-estimating-equations extension of logistic regression, with an exchangeable covariance matrix, to account for the clustering of women within hospitals [68]. Changes in the risk of major perinatal morbidity in the two study groups between the 1-year baseline (pre-intervention) period and the 2-year intervention period will be compared with the use of an adjusted odds ratio (with 95\% confidence intervals) for the interaction between group (intervention vs. control) and time period (intervention vs. baseline) [64, 65]. The adjusted odds ratio for interaction will be estimated with the use of data on women who were delivered during the baseline period or the intervention period and measures the intervention effect with the difference-in-differences approach [69, 70], which is adapted for generalized-estimating equation analyses of clustered binary outcomes [65]. In each group, the change over time, from the baseline to the intervention period, will be measured by the respective odds ratio, i.e. the exponent of the difference between log odds of cesarean delivery in intervention versus baseline periods. Then, additional change over time in the intervention group, relative to the concurrent change in the control group, will be estimated by the difference between group-specific differences. The exponent of the resulting difference-in-differences in log odds provides the adjusted odds ratio, with 95\% CIs, for the interaction between group (intervention vs. control) and time (intervention vs. baseline), which will be used as the main measure of the intervention effect $[64,69,70]$. Two-tailed $P$ values of less than 0.05 will be considered to indicate statistical significance.

All primary analyses will include adjustments for prespecified potential maternal, perinatal and institutional risk factors associated with major perinatal morbidity [64]. To conform to the intention-to-treat approach, all eligible women who deliver at participating hospitals will be included in the analyses. For adjustment variables for which less than $1 \%$ of the data are missing, we will use random imputation (performed on the basis of the observed distributions of the imputed variable and of highly correlated covariates). Variables for which more than $1 \%$ of the data are missing will be excluded from the analyses. Randomization will be stratified by level of care in hospitals. To assess whether the intervention effect will vary according to the level of care, we will test the corresponding three-way interactions: level of care $\times$ intervention $\times$ time. Subgroup-specific intervention effects will be reported for outcomes with significant three-way interactions (two-tailed tests, with $P$ value of less than 0.05 will be considered statistically significant) $[69,70]$.

Secondary outcomes will be analyzed by means of methods similar to those used for the primary outcome. Subgroup analyses for preterm and term birth will be also planned to take into account major neonatal morbidity related to preterm birth and not related to the mode of delivery. If the generalized-estimating- 
equations models do not converge, the intervention effect will be estimated with the use of multivariable logistic model, which do not account for within-hospital clustering; to correct for the resulting underestimation of the standard errors, a conservative $P$ value of less than 0.001 will be used [68, 70]. All analyses will be performed with the use of SAS software, version 9.4 by an independent team whose members will be unaware of the group assignments.

\section{Economic evaluation}

Cost-effectiveness analyses will take the perspective of the publicly funded health care system [71]. The time horizon will capture hospital-based costs and clinical events for mothers and neonates from labour onset to a minimum of 3 months postpartum. Resource use will be identified and measured from patient charts and valued using standardised government sources, following recommended methods for costing in the Canadian context [72]. A discount rate of $5 \%$ will be applied, and results will be compared to two scenarios implementing alternative discount rates of 3\% to facilitate comparisons with other jurisdictions and $0 \%$ to explore the effect of discounting [71].

The main analysis will be an ITT analysis including all trial participants. To assess intervention impact, we will model changes in a composite index of major perinatal morbidities and costs in the two study groups between the 1-year baseline period and the 2-year intervention period using adjusted regression coefficients (with 95\% confidence intervals) for the interaction between group (intervention or control) and time period (intervention or baseline) $[64,70]$. Costs and effects will be modeled jointly using bivariate multilevel linear models that explicitly recognize potential correlations between the bivariate outcomes (major perinatal morbidities and costs) at individual and cluster (hospital) levels [73]. Crude and adjusted statistical models will be also estimated. Crude models will include study group (intervention vs. control), time period (baseline vs. intervention) and the interaction between study groups and time period. Adjusted models will use precisely the same variables for adjustment of confounding, as the main trial. All variable definitions will be identical to those used for the main trial. To address potential heterogeneity in costeffectiveness, analyses will be repeated for pre-specified subgroups of interest (level of care, prematurity).

For each analysis, the incremental cost-effectiveness ratio (ICER) statistic will be calculated as the ratio of additional costs per reduction in major perinatal morbidities, and estimate its confidence interval using the Bayesian Markov Chain Monte Carlo (MCMC) method [74]. As interpretation of the ICER can be challenging, the Incremental Net monetary Benefit (INB) will be also calculated using a range of reasonable values to represent societal willingness to pay $(\Lambda)[75,76]$. Analyses will be also conducted to explore the implications of stochastic, parameter and structural uncertainty, as well as heterogeneity [77]. We will use the Bayesian MCMC method to ascertain the joint uncertainty of the estimands, incremental costs and effects [78], and costeffectiveness planes plotting the values of incremental costs and effects stored in the Markov chains to present the joint uncertainty of the estimands [74]. To explore potential heterogeneity we will repeat all analyses over subgroups based on hospital level of care [74]. Results of the uncertainty analyses will be graphically presented in a cost-effectiveness acceptability curve (CEAC) that depicts the uncertainty about the new intervention being cost-effective as a function of societal willingness to pay (^) [77-80]. Finally, we will decompose costs into categories reflecting the secondary trial outcomes to identify drivers of change. Cost-effectiveness models will be implemented in MLwiN [81] within a Stata environment [82]. An independent team not involved in the conduct or analysis of the main trial will perform the economic analyses.

\section{Potential limitations of the trial}

Our study could have some limitations. Since hospitals will be the unit of randomization, and the number of deliveries varies across hospitals, there could be differences in the distribution of hospital characteristics across groups at baseline. These differences will be adjusted a priori in multivariable analyses. Because of potential intra-cluster variation among hospitals, the trial results could be driven by a few hospitals. Consequently, adjusted, center-specific multivariable analyses will be also conducted to assess changes over time in the major perinatal morbidity rates in each individual hospital. Furthermore, the intervention may not be fully implemented in each intervention hospitals. Finally, because we will assess a complex, multifaceted intervention, it will not be possible to determine which of its components will be primarily responsible for any observed effect.

\section{Ethical issues}

\section{Ethics committee approval}

The trial has been approved by the Ethics Committee of the CHU de Québec-Université Laval in Québec, Canada and of all 40 participating hospitals (MP-20-2016-2718; MP-20-2017-3220). The PRISMA trial is registered on the ISRCTN registry under the number ISRCTN15346559 (http://www.isrctn.com/). The results of the trial will be reported according to the CONSORT Statement (SPIRIT Checklist, Additional file 1). 


\section{Information and consent forms}

The participating hospitals are included on the basis of an informed consent form signed by the head of obstetrics. The informed consent form specifies that: (1) the site can withdraw from the program at any time; (2) the data collection will continue until the end of the trial, even if a site withdraw from the project; and 93) that the PRISMA program will be offered to control group hospitals at the end of the trial if the intervention is proven effective. Collection of clinical data from medical records is authorized by the director of professional services in each participating hospital and does not require patient consent. During the intervention period, the involvement of patients will be sought with the use of the decision analysis tool (DAT) and for the LUS thickness measurement. The DAT includes an implied consent which states that filling it and returning it to their physician represent a women's consent to use the DAT. The authorisation for an additional ultrasound measurement (LUS thickness) will be obtained by a written information and consent form.

\section{Interim analyses and stopping rules}

An independent Data Safety and Monitoring Board (DSMB) has been established, made up of three international experts in epidemiology and biostatistics, reproductive health research, and obstetrics and gynaecology. It will be responsible for ensuring the safety of the trial and for monitoring the progress of the research according to the protocol. Serious adverse events (maternal and perinatal death, perinatal asphyxia, hemostatic, hysterectomy, uterine rupture) will be monitored annually during the intervention by the DSMB, blinded, for all patients in the study. To assess the effectiveness of the program at the end of the first year of the intervention period, an intermediate analysis will be planned using Peto criteria $(\alpha=0.001)$, which ensure that a total type-I risk of error, for the final analysis, of 0.05. The DSMB may decide to stop the trial for the following reasons: detection of adverse events, poor data quality, low levels of implementation, high contamination in the control group, fraud, and new information that would rule the trial unnecessary, futile, or unethical.

\section{Sponsor and project administration}

The project was approved by the Canadian Institutes of Health Research (CIHR) and funded in July 2015. The trial begun in April 2016. The funds are managed by the Université Laval. The multidisciplinary PRISMA research group is composed of researchers and physicians from Canada, United States and France with an international expertise in maternal and perinatal health, VBAC, clinical research, RCTs, biostatistics, improvement of quality of care and assessment of health care programs. The PRISMA intervention program is coordinated by a group of experts in maternal health and VBAC, in collaboration with the Society of Obstetricians and Gynaecologists of Canada (SOGC), the Ministère de la santé et des Services Sociaux du Québec (MSSSQ), the Association des Obstétriciens Gynécologues du Québec (AOGQ), the Association des Omnipraticiens en Périnatalité du Québec (AOPQ), and the Canadian Association of Perinatal and Women's Health Nurses (CAPWHN). The management of the program implementation in hospitals of the intervention group and the conduct of mid-intervention audits will be carried out by the administrative coordinator of the trial. The data management and quality control are assumed by the scientific coordinator, who is also responsible for sending data to the DSMB for annual assessment of the safety of the trial and compliance with the protocol and timeline. The data collection and the intervention are two independent processes. A cross-disciplinary Steering Committee, with extensive international expertise in RCT, VBAC, quality of care improvement and health care program assessment, meets at least every 3 months to coordinate the trial, taking into account DSMB recommendations.

\section{Discussion}

The PRISMA trial is a phase III cluster randomized controlled trial to assess the effectiveness of a multifaceted intervention aiming to reduce severe perinatal morbidity in women with one previous cesarean delivery. The PRISMA Program is designed to facilitate: 1) women's decision-making process, using a decision analysis tool (DAT); 2) an estimate of uterine rupture risk during TOL using ultrasound evaluation of low-uterine segment thickness; 3) an estimate of chance of TOL success, using a validated prediction tool; and 4) the implementation of best practices for intrapartum management.

The DAT will be available in each intervention hospital in the clinic waiting rooms and distributed by the treating physician early during the pregnancy to help women make an enlightened choice. This tool will be also discussed with the physician during the next appointment. An onsite professional training program from the SOGC including decisional algorithms about selection of women for a TOL, chance of VBAC success, optimal management of delivery and labor dystocia among women with one prior cesarean delivery, is also planned in each intervention hospital. This professional training will be planned several times in each hospital in order to be able to train a high number of health professionals (obstetricians and gynaecologist, family physician, nurses, midwives). Finally, several onsite ultrasound training are planned in each intervention hospital, during the 5-month implementation period, in order to 
train ultrasound health professionals to assess the risk of uterine rupture at 35 weeks (LUS technic) among women with one prior cesarean delivery.

An important challenge relies in the implementation of the program, which may differ in different hospitals. In this Trial, a high level of implementation will be ensured by the support of local partners and healthcare professionals identified in intervention hospitals, by continuous internal assessments for compliance, by an annual site monitoring visit or more if required, and by a centralized platform in Québec allowing an external validation of each LUS thickness measures. This real time validation will ensure a constant follow up with participating physicians.

The PRIMSA Program aims to provide the right intervention for the right woman at the right time, and will contribute to reduce the perinatal morbidity among women with a prior cesarean delivery. This trial will provide a high level of evidence regarding the choice of the optimal mode of delivery, as well as the management of delivery in women with a prior cesarean.

\section{Trial status}

At the time of submission, participating hospital have been enrolled, randomized and patient recruitment begun in one hospital.

\section{Additional file}

Additional file 1: SPIRIT 2013 Checklist: Recommended items to address in a clinical trial protocol and related documents. (PDF $100 \mathrm{~kb}$ )

\section{Acknowledgments}

We wish to thank the Canadian Institutes of Health Research (CIHR), our sponsor, which supports the PRISMA trial, grant number 201503MOP-340680RC1-CFBA-153236.

PRISMA Trial research group: Laval University (Quebec, Canada): Nils Chaillet, Emmanuel Bujold, Suzanne Demers, Marylène Dugas, Bruno Piedboeuf; University of Montreal (Montreal, Canada): Mira Johri, François Beaudoin, Robert Gauthier, Benoit Masse, François Audibert, Louise Duperron, Marie Josée Bédard, François Champagne, Diane Francoeur; Sherbrooke University (Sherbrooke, Canada): William D. Fraser, Jean-Charles Pasquier; McGill University (Montreal, Canada): Haim Abenhaim, Robert Gagnon, Patricia Monnier, Isabelle Girard, Guy-Paul Gagné; Northwestern University (Chicago, IL, USA): William A. Grobman; University of Alabama (Birmingham, Alabama, USA): Allison Shorten: Versailles University (Paris, France): Patrick Rozenberg; Society of Obstetricians and Gynaecologists of Canada (Ottawa, Canada): Jennifer Blake; Association des Obstétriciens et Gynécologues du Québec (Montréal, Canada): Isabelle Girard; Fédération des médecins spécialistes du Québec (Québec, Canada): Diane Francoeur; Association des Omnipraticiens en Périnatalité du Québec (Montréal, Canada): Andrée Gagnon; Canadian Association of Perinatal and Women's Health Nurses (Ottawa, Canada): Luisa Ciofani; Ordre des Sages-Femmes du Québec (Montréal, Canada): Marie-Eve St Laurent; and Ministry of Health and Social Services (Québec, Canada): Daniel Riverin. Statistical Analyses: Benoit Masse. Coordination and Data Management: Eric Dubé, Simon Blouin. Data Safety and Monitoring Board: Robert Platt (Biostatistiques, University McGill, Montreal, QC, Canada), Shiliang Liu (Public Health Agency of Canada, Ottawa, Canada) et Fernando Althabe (Latin American Center for Perinatology and Human Development, Buenos Aires University, Brazil).

\section{Funding}

The PRISMA trial is funded by the Canadian Institutes of Health Research (CIHR, funding number MOP-142448). The sponsor of the study had no role in study design or writing of the protocol. The corresponding author had final responsibility for the decision to submit for publication.

Availability of data and materials

Not applicable.

\section{Authors' contributions}

$\mathrm{NC}$ and $\mathrm{EB}$ are PI on the grant and are responsible for conception and design of study, drafting manuscript, and critical revision of manuscript. BM, WAG, PR, JCP, AS, MJ, FB, HA, SD, WF and MD were involved in conception and design of study. BM developed the statistical analysis plan. EB, SD, PR and RG especially developed the LUS technic. WAG developed the Grobman nomogram. AS and MD developed and adapted the decision analysis tool. MJ worked on the economic evaluation. SB and ED are study coordinators and contributed to the writing of the manuscript. All authors revised and approved the final manuscript.

\section{Ethics approval and consent to participate}

The trial has been approved by the ethics committee of the $\mathrm{CHU}$ de Québec-Université Laval in Québec, Canada and of all 40 participating hospitals (MP-20-2016-2718; MP-20-2017-3220). Collection of clinical data from medical records is authorized by the Director of Professional Services in each participating hospital. The authorisation for an additional ultrasound measurement (LUS thickness) will be obtained by a written information and consent form.

\section{Consent for publication}

All authors have consented to publication of this article.

\section{Competing interests}

The authors declare that they have no competing interests.

\section{Publisher's Note}

Springer Nature remains neutral with regard to jurisdictional claims in published maps and institutional affiliations.

\section{Author details}

'Department of Obstetrics and Gynaecology, Laval University, Quebec, QC, Canada. ${ }^{2}$ Department of Epidemiology and Biostatistics, University of Montréal, Montréal, QC, Canada. ${ }^{3}$ Department of Obstetrics and Gynaecology, Northwestern University, Chicago, IL, USA. ${ }^{4}$ Service de gynécologie obstétrique et médecine de la reproduction, Centre hospitalier intercommunal de Poissy/Saint-Germain-en-Laye, 10, rue du Champ-Gaillard, 78303 Poissy, France. ${ }^{5}$ Department of Obstetrics and Gynecology, Sherbrooke University, Quebec, QC, Canada. ${ }^{6}$ UAB School of Nursing, University of Alabama, Birmingham, AL, USA. University of Montreal, Hospital Research. Center (CRCHUM), Montreal, QC, Canada. ${ }^{8}$ Department of Obstetrics and Gynecology, University of Montreal, Montreal, QC, Canada. ${ }^{9}$ Department of Obstetrics and Gynecology, McGill University, Jewish Hospital, Montreal, QC, Canada. ${ }^{10}$ Population Health and Optimal Health Practices Research Unit, CHU de Québec Research Centre, Quebec, QC, Canada. ${ }^{11}$ Faculté de Médecine, Département d'Obstétrique \& Gynécologie, Université Laval, Centre de recherche du CHUQ, 2705, Boul. Laurier, local T-R-92, Quebec, QC G1V 4G2, Canada.

Received: 22 March 2017 Accepted: 15 August 2017

Published online: 20 September 2017

\section{References}

1. Public Health Agency of Canada. Canadian perinatal health report. Ottawa; 2008

2. Health system performance: health indicators. Ottawa: $\mathrm{ClH}, 2005$.

3. Canadian Institute for Health Information. Giving birth in Canada: regional trends from 2001-2002 to 2005-2006. Ottawa; 2007.

4. Allen VM, O'Connell CM, Liston RM, Baskett TF. Maternal morbidity associated with cesarean delivery without labor compared with spontaneous onset of labor at term. Obstet Gynecol. 2003;102:477-82. 
5. Zanardo V, Simbi AK, Franzoi M, Solda G, Salvadori A, Trevisanuto D. Neonatal respiratory morbidity risk and mode of delivery at term: influence of timing of elective caesarean delivery. Acta Paediatr. 2004;93:643-7.

6. Martin JA, Hamilton BE, Sutton PD, Ventura SJ, Menacker F, Munson ML Births: final data for 2003. Natl Vital Stat Rep. 2005;54:1-116.

7. Levine EM, Ghai V, Barton JJ, Strom CM. Mode of delivery and risk of respiratory diseases in newborns. Obstet Gynecol. 2001;97:439-42.

8. Canadian Institute for Health Information. Giving birth in Canada: the costs Ottawa; 2007

9. Rowe AK, de Savigny D, Lanata CF, Victora CG. How can we achieve and maintain high-quality performance of health workers in low-resource settings? Lancet. 2005;366:1026-35.

10. Martin JA, Hamilton BE, Sutton PD, et al. Births: final data for 2005. Natl Vital Stat Rep. 2007;56:1-103.

11. Guise JM, McDonagh MS, Osterweil P, Nygren P, Chan BK, Helfand M. Systematic review of the incidence and consequences of uterine rupture in women with previous caesarean section. BMJ. 2004;329:19-25.

12. Bujold E, Bujold C, Hamilton EF, Harel F, Gauthier RJ. The impact of a singlelayer or double-layer closure on uterine rupture. Am J Obstet Gynecol. 2002; 186:1326-30.

13. McMahon MJ, Luther ER, Bowes Jr WA, Olshan AF. Comparison of a trial of labor with an elective second cesarean section. N Engl J Med. 1996;335:689-95.

14. Pridjian G. Labor after prior cesarean section. Clin Obstet Gynecol. 1992;35:445-56.

15. Lydon-Rochelle M, Holt VL, Easterling TR, Martin DP. Risk of uterine rupture during labor among women with a prior cesarean delivery. N Engl J Med. 2001;345:3-8.

16. Guise JM, Eden $\mathrm{K}$, Emeis $\mathrm{C}$, et al. Vaginal birth after cesarean: new insights. Evid Rep Technol Assess (Full Rep). 2010;191:1-397.

17. Liu S, Liston RM, Joseph KS, et al. Maternal mortality and severe morbidity associated with low-risk planned cesarean delivery versus planned vaginal delivery at term. CMAJ. 2007;176:455-60.

18. Wen SW, Rusen ID, Walker M, et al. Comparison of maternal mortality and morbidity between trial of labor and elective cesarean section among women with previous cesarean delivery. Am J Obstet Gynecol. 2004;191:1263-9.

19. Penna L, Arulkumaran S. Cesarean section for non-medical reasons. Int J Gynaecol Obstet. 2003;82:399-409.

20. American College of Obstetricians and Gynecologists, Task Force on Caesarean Delivery Rates. Evaluation of Caesarean delivery. Washington (DC): American College of Obstetricians and Gynecologists; 2000.

21. Meikle SF, Steiner CA, Zhang J, Lawrence WL. A national estimate of the elective primary cesarean delivery rate. Obstet Gynecol. 2005;105:751-6.

22. Greene MF. Vaginal delivery after cesarean section-is the risk acceptable? N Engl J Med. 2001;345:54-5.

23. Greene MF. Vaginal birth after cesarean revisited. N Engl J Med. 2004;351:2647-9.

24. Dugas M, Shorten A, Dube E, Wassef M, Bujold E, Chaillet N. Decision aid tools to support women's decision making in pregnancy and birth: a systematic review and meta-analysis. Soc Sci Med. 2012;74:1968-78.

25. Grobman WA, Lai Y, Landon MB, et al. Development of a nomogram for prediction of vaginal birth after cesarean delivery. Obstet Gynecol. 2007;109: 806-12

26. Grobman WA, Lai Y, Landon MB, et al. Does information available at admission for delivery improve prediction of vaginal birth after cesarean? Am J Perinatol. 2009;26:693-701.

27. Chaillet N, Bujold E, Dube E, Grobman WA. Validation of a prediction model for vaginal birth after caesarean. J Obstet Gynaecol Can. 2013;35:119-24.

28. Bujold E, Jastrow N, Simoneau J, Brunet S, Gauthier RJ. Prediction of complete uterine rupture by sonographic evaluation of the lower uterine segment. Am J Obstet Gynecol. 2009;201(3):320. e1-6.

29. Jastrow $N$, Chaillet $N$, Roberge $S$, Morency AM, Lacasse $Y$, Bujold E. Sonographic lower uterine segment thickness and risk of uterine scar defect: a systematic review. J Obstet Gynaecol Can. 2010;32:321-7.

30. Bujold E, Jastrow N, Gauthier RJ, et al. Measurement of the lower uterine segment: are we ready for a clinical application? J Obstet Gynaecol Can. 2010:32:339-40.

31. Montgomery AA, Emmett CL, Fahey T, et al. Two decision aids for mode of delivery among women with previous caesarean section: randomised controlled trial. BMJ. 2007;334:1305

32. Frost J, Shaw A, Montgomery A, Murphy DJ. Women's views on the use of decision aids for decision making about the method of delivery following a previous caesarean section: qualitative interview study. BJOG. 2009;116:896-905
33. Glazier R, Goel V, Holzapfel S, Summers A, Pugh P, Yeung M. Written patient information about triple-marker screening: a randomized, controlled trial. Obstet Gynecol. 1997;90:769-74.

34. O'Cathain A, Walters SJ, Nicholl JP, Thomas KJ, Kirkham M. Use of evidence based leaflets to promote informed choice in maternity care: randomised controlled trial in everyday practice. BMJ. 2002;324:643.

35. Stewart A, Sodhi V, Harper N, Yentis SM. Assessment of the effect upon maternal knowledge of an information leaflet about pain relief in labour. Anaesthesia. 2003:58:1015-9.

36. Wong SS, Thornton JG, Gbolade B, Bekker HL. A randomised controlled trial of a decision-aid leaflet to facilitate women's choice between pregnancy termination methods. BJOG. 2006;113:688-94.

37. Hunter AG, Cappelli M, Humphreys L, et al. A randomized trial comparing alternative approaches to prenatal diagnosis counseling in advanced maternal age patients. Clin Genet. 2005:67:303-13.

38. Secker-Walker RH, Solomon LJ, Flynn BS, Skelly JM, Mead PB. Reducing smoking during pregnancy and postpartum: physician's advice supported by individual counseling. Prev Med. 1998;27:422-30.

39. Thornton JG, Hewison J, Lilford RJ, Vail A. A randomised trial of three methods of giving information about prenatal testing. BMJ. 1995;311: 1127-30.

40. Bekker HL, Hewison J, Thornton JG. Applying decision analysis to facilitate informed decision making about prenatal diagnosis for Down syndrome: a randomised controlled trial. Prenat Diagn. 2004;24:265-75.

41. Kuppermann M, Norton ME, Gates E, et al. Computerized prenatal genetic testing decision-assisting tool: a randomized controlled trial. Obstet Gynecol. 2009;113:53-63.

42. Nassar N, Roberts $\mathrm{CL}$, Raynes-Greenow CH, Barratt A, Peat B, Decision Aid for Breech Presentation Trial C. Evaluation of a decision aid for women with breech presentation at term: a randomised controlled trial [ISRCTN14570598]. BJOG. 2007;114:325-33.

43. Shorten A, Shorten B, Keogh J, West S, Morris J. Making choices for childbirth: a randomized controlled trial of a decision-aid for informed birth after cesarean. Birth. 2005:32:252-61.

44. O'Connor AM, Stacey D, Entwistle V, et al. Decision aids for people facing health treatment or screening decisions. Cochrane Database Syst Rev. 2003, Issue 1:CD001431.

45. Bujold E, Gauthier RJ. Neonatal morbidity associated with uterine rupture: what are the risk factors? Am J Obstet Gynecol. 2002;186:311-4.

46. Costantine MM, Fox $\mathrm{K}$, Byers $\mathrm{BD}$, et al. Validation of the prediction model for success of vaginal birth after cesarean delivery. Obstet Gynecol. 2009; 114:1029-33.

47. Costantine MM, Fox KA, Pacheco LD, et al. Does information available at delivery improve the accuracy of predicting vaginal birth after cesarean? Validation of the published models in an independent patient cohort. Am J Perinatol. 2011;28:293-8.

48. Grobman WA, Lai Y, Landon MB, et al. Can a prediction model for vaginal birth after cesarean also predict the probability of morbidity related to a trial of labor? Am J Obstet Gynecol. 2009:200:56. e1-6.

49. Jastrow N, Demers $\mathrm{S}$, Chaillet N, et al. Lower uterine segment thickness to prevent uterine rupture and adverse perinatal outcomes: a multicenter prospective study. Am J Obstet Gynecol. 2016;215(5):604. e1-6.

50. Boutin A, Jastrow N, Girard M, et al. Reliability of two-dimensional transvaginal sonographic measurement of lower uterine segment thickness using video sequences. Am J Perinatol. 2012;29(07):527-32.

51. Boutin A, Jastrow N, Roberge $\mathrm{S}$, et al. Reliability of 3-dimensional transvaginal sonographic measurement of lower uterine segment thickness, J Ultrasound Med. 2012;31:933-9.

52. Lavender T, Hart A, Smyth RM. Effect of partogram use on outcomes for women in spontaneous labour at term. Cochrane Database Syst Rev. 2013 CD005461.

53. Society of Obstetrician and Gynaecologist of Canada. Managing obstetric risk efficiently. 2004

54. Johri M, Damschroder $\amalg$, Zikmund-Fisher BJ, Ubel PA. The importance of age in allocating health care resources: does intervention-type matter? Health Econ. 2005:14:669-78.

55. Ottawa Decision Support Framework, Patient decision aids. Ottawa Hospital Research Institute (OHRI). 2009. (https://decisionaid.ohri.ca/methods.html. Accessed 15 Dec 2011).

56. International Patient Decision AID Standard (IPDAS) http://ipdas.ohri.ca/. Accessed 15 Dec 2011 
57. Sen S, Malik S, Salhan S. Ultrasonographic evaluation of lower uterine segment thickness in patients of previous cesarean section. Int J Gynaecol Obstet. 2004;87:215-9.

58. Martins WP, Barra DA, Gallarreta FM, Nastri CO, Filho FM. Lower uterine segment thickness measurement in pregnant women with previous Cesarean section: reliability analysis using two- and three-dimensional transabdominal and transvaginal ultrasound. Ultrasound Obstet Gynecol. 2009:33:301-6.

59. Bujold E, Girard M, Jastrow N, Brassard N. OP15.08: Comparison between transvaginal and abdominal sonography of lower uterine segment thickness near term. Ultrasound Obstet Gynecol. 2009:34:109-10.

60. Bujold $E$, Francoeur D. Neonatal morbidity and decision-delivery interval in patients with uterine rupture. J Obstet Gynaecol Can. 2005;27:671-3. author reply 3 .

61. Guise JM, Berlin M, McDonagh M, Osterweil P, Chan B, Helfand M. Safety of vaginal birth after cesarean: a systematic review. Obstet Gynecol. 2004;103: 420-9.

62. Guise JM, Hashima J, Osterweil P. Evidence-based vaginal birth after Caesarean section. Best Pract Res Clin Obstet Gynaecol. 2005;19:117-30.

63. DACIMA Clinical Suite. (http://www.dacimasoftware.com/pdf/Dacima Clinical_2016.pdf. Accessed Dec 2016)

64. Chaillet N, Dumont A, Abrahamowicz M, et al. A cluster-randomized trial to reduce cesarean delivery rates in Quebec. N Engl J Med. 2015;372:1710-21.

65. Donner A, Klar N. Design and analysis of cluster randomization trials in health research. New York: Oxford University Press; 2000.

66. Pinol A, Piaggio G. ACluster: design and analysis of cluster randomization trials. 2nd ed. Geneva: Worl Health Organization; 2000.

67. Althabe F, Belizan JM, Villar J, et al. Mandatory second opinion to reduce rates of unnecessary caesarean sections in Latin America: a cluster randomised controlled trial. Lancet. 2004;363:1934-40.

68. Zeger SL, Liang KY, Albert PS. Models for longitudinal data: a generalized estimating equation approach. Biometrics. 1988;44:1049-60.

69. Abrahamowicz M, Beauchamp ME, Fournier P. Dumont A. Evidence of subgroup-specific treatment effect in the absence of an overall effect: is there really a contradiction? Pharmacoepidemiol Drug Saf. 2013;22:1178-88.

70. Dumont A, Fournier P, Abrahamowicz M, et al. Quality of care, risk management, and technology in obstetrics to reduce hospital-based maternal mortality in Senegal and Mali (QUARITE): a cluster-randomised trial. Lancet. 2013;382:146-57.

71. Canadian Agency for Drugs and Technologies in Health (CADTH). Guidelines for the Economic Evaluation of Health Technologies. Canada; 2006.

72. Guidance document for the costing of health care resources in the Canadian setting: 2nd edition. Ottawa: CADTH; 2016.

73. Gomes M, Grieve R, Nixon R, Edmunds WJ. Statistical methods for costeffectiveness analyses that use data from cluster randomized trials: a systematic review and checklist for critical appraisal. Med Decis Making. 2012;32:209-20.

74. O'Brien BJ, Briggs AH. Analysis of uncertainty in health care costeffectiveness studies: an introduction to statistical issues and methods. StatMethods MedRes. 2002;11:455-68.

75. Hoch JS, Briggs AH, Willan AR. Something old, something new, something borrowed, something blue: a framework for the marriage of health econometrics and cost-effectiveness analysis. Health Econ. 2002;11:415-30

76. Stinnett AA, Mullahy J. Net health benefits: a new framework for the analysis of uncertainty in cost-effectiveness analysis [see comments]. Med Decis Making. 1998;18:568-80.

77. Briggs AH, Weinstein MC, Fenwick EA, et al. Model parameter estimation and uncertainty analysis: a report of the ISPOR-SMDM Modeling Good Research Practices Task Force Working Group-6. Med Decis Making. 2012;32:722-32.

78. Browne WJ. MCMC Estimation in MLwiN, v2.26. Bristol, UK: Centre for Multilevel Modelling, University of Bristol; 2012.

79. Fenwick E, Claxton K, Sculpher M. Representing uncertainty: the role of costeffectiveness acceptability curves. Health Econ. 2001;10:779-87.

80. Fenwick E, O'Brien BJ, Briggs A. Cost-effectiveness acceptability curves-facts, fallacies and frequently asked questions. Health Econ. 2004;13:405-15.

81. Rasbash J, Charlton C, Browne WJ, Healy M, Cameron B. MLwiN Version 2. 10. Centre for Multilevel Modelling, University of Bristol; 2009.

82. Stata 14.1. College Station, TX: Stata Corp; 2015.

\section{Submit your next manuscript to BioMed Central and we will help you at every step:}

- We accept pre-submission inquiries

- Our selector tool helps you to find the most relevant journal

- We provide round the clock customer support

- Convenient online submission

- Thorough peer review

- Inclusion in PubMed and all major indexing services

- Maximum visibility for your research

Submit your manuscript at www.biomedcentral.com/submit

Biomed Central 